\title{
Alterations of differential gene expression during the morphogenesis induction in wheat tissue culture (transcriptome analysis)
}

\author{
Bishimbayeva N.K. ${ }^{1,2 *}$, Begzat A.N. ${ }^{1}$, Mitra A. ${ }^{3}$, Kairov U. ${ }^{4}$, Nakisbekov N.O. ${ }^{5}$, \\ Molkenov A. ${ }^{4}$, Amirbekov A.S. ${ }^{5}$, Li Ch. ${ }^{6}$, Huang K. ${ }^{6}$, Rakhimbayev I.R. ${ }^{7}$ \\ ${ }^{1}$ Al-Farabi Kazakh National University, Almaty, Kazakhstan \\ ${ }^{2}$ Kazakh National Agrarian University, Almaty, Kazakhstan \\ ${ }^{3}$ University of Nebraska-Lincoln, Nebraska, USA \\ ${ }^{4}$ Nazarbayev University, Nur-Sultan, Kazakhstan \\ ${ }^{5}$ Asfendiarov Kazakh National Medical University, Almaty, Kazakhstan \\ ${ }^{6}$ Beijing Genomics Institute, Shenzhen, China \\ ${ }^{7}$ Institute of Plant Biology and Biotechnology, Almaty, Kazakhstan \\ *e-mail:gen_jan@mail.ru
}

Today, the most modern approach for study the gene expression is profiling of the entire transcriptome. The cell identity is defined by its transcriptome, i. e., by a complete set of expressed RNA transcripts. Profiling of the whole transcriptome is widely used to assess the relative gene expression in cells, tissues, organisms, or under different conditions. The main goal of this investigation is identification of differentially expressed genes by whole-transcriptome sequencing of wheat cell culture samples during the induction of long-term embryogenic cell lines. For this purpose the transcriptomes of 7-days wheat calli induced for long-term embryodogenesis and control uninduced tissues have been compared. Initial globular non morphogenic callus, subcultivated on MS nutrient media with 2,4-D have been used as a control uninduced sample. Control variants were compared with globular callus, transferred for the induction of long-term embryogenic cell lines on MS media with 2,4-D and high (stress) concentration of mineral salts. As a result of transcriptome RNAseq analysis, key genes involved in early stages of induction of long-term embryogenic potential have been identified. 974 genes with up-regulated expression have been established belonging to the categories of the protein proteolysis and cellular proteins involved in catabolism, 419 of them were recognized. 834 genes with down-regulated expression have been revealed belonging to the categories of response factors for signals, 403 of them were recognized.

Acknowledgements: This work performed in the framework of fundamental research program No. 0149, Target Program Funding, IPBB, Science Committee, Ministry of Education and Science, Republic of Kazakhstan (2015-2017). 